\title{
Tensor products of modal logics
}

\author{
I. Shapirovsky and V. Shehtman* \\ Institute for Information Transmission Problems, Moscow, Russia.
}

Products were introduced in the 1970s as a natural type of combined modal logics. They arise in different areas of pure and applied logics - spatial reasoning, multi-agent systems, quantified modal and intuitionistic logics etc. The theory of products was systematized and essentially developed first in the paper GS98 and then in the book GKWZ03, but during the past 10 years new important results were proved and the research is going on, cf. Kur07.

Recall that the product of modal logics is defined as the logic of the class of products of their Kripke frames. On the one hand, this definition is quite natural, and in some cases products can be simply axiomatized and have nice properties. On the other hand, products are always Kripke complete. However, Kripke semantics sometimes may be inadequate. So different logics $\mathrm{L}_{1}, \mathrm{~L}_{1}^{\prime}$ can have the same frames; then $\mathrm{L}_{1} \times \mathrm{L}_{2}=\mathrm{L}_{1}^{\prime} \times \mathrm{L}_{2}$ for any $\mathrm{L}_{2}$ - which looks strange. Another peculiarity is logical non-invariance: it may happen that for some frames $\log (F)=\log \left(\mathrm{F}^{\prime}\right)$, while $\log (F \times G) \neq \log \left(F^{\prime} \times G\right)$. Also, if logics $L_{1}$ and $L_{2}$ are consistent, but $L_{1}$ has an empty class of frames, then $\mathrm{L}_{1} \times \mathrm{L}_{2}$ is inconsistent.

To amend the situation, we can try to define products of Kripke of models (or, equivalently, general Kripke frames or modal algebras). The following problem was mentioned in Kur07, p. 877]: There are several attempts for extending the product construction from Kripke complete logics to arbitrary modal logics, mainly by considering product-like constructions on Kripke models. All the suggested methods so far result in sets of formulas that are not closed under the rule of Substitution.

Nevertheless, by that time a possible answer already existed (but was unnoticed): it was given by Yasusi Hasimoto in [Has00, who introduced so-called shifted products of modal algebras. In this paper we show that a shifted product acts on Boolean algebras exactly as a tensor product (an observation first made by Dov Gabbay [GSS]). So now we call this operation a tensor product of modal algebras. We also propose a filtration technique for models based on tensor products and obtain some decidability results.

\section{Tensor products and chequered valuations}

It is well known that we can regard every Boolean algebra as a Boolean ring. Every Boolean ring is a commutative algebra with an idempotent multiplication over the two-element field $\mathbf{F}_{2}$. So the standard tensor product construction is applicable here [AM69].

Viz., the tensor product of algebras $A, B$ is the pair $(\pi, A \otimes B)$, where $\pi:(a, b) \mapsto a \otimes b$ is a bilinear map $A \times B \rightarrow A \otimes B$ such that every bilinear $f: A \times B \rightarrow C$ uniquely factors through $\pi$, i.e., $f=g \cdot \pi$ for a unique linear $g$. The multiplication in $A \otimes B$ is defined in such a way that $(a \otimes b)(c \otimes d)=a c \otimes b d$.

Proposition 1.1. The tensor product of Boolean rings is a Boolean ring.

Our aim is to define tensor products of modal algebras. To this end, let us describe the tensor product construction for Boolean rings more explicitly.

${ }^{*}$ The work on this paper was supported by the Russian Foundation for Basic Research (projects no. 11-0193107 and 11-01-00958), and the Russia-Israel Scientific Research Cooperation, project no. 206891.

N. Galatos, A. Kurz, C. Tsinakis (eds.), TACL 2013 (EPiC Series, vol. 25), pp. 199-203 
Definition 1.2. A set $U \times V$, where $U \subseteq X, V \subseteq Y$, is called a rectangle in $X \times Y$. A chequered subset of $X \times Y$ is a finite union of rectangles.

Proposition 1.3. Has00] The set of all chequered subsets of $W_{1} \times W_{2}$ is closed under Boolean operations. Moreover, if $A_{i}$ is a subalgebra of $2^{W_{i}}, i=1,2$, then the set of all finite unions of rectangles $V_{1} \times V_{2}$, where $V_{i} \in A_{i}$, is closed under Boolean operations as well.

For nonempty sets $X, Y$ let $\operatorname{ch}(X, Y)$ be the Boolean algebra of all chequered subsets of $X \times Y$.

Theorem 1.4. GSS Let $X, Y$ be nonempty sets, $A, B$ subalgebras of $2^{X}, 2^{Y}$ respectively, $C$ the Boolean algebra of finite unions of rectangles $U \times V$, where $U \in A, V \in B$. Consider the map $\pi: 2^{X} \times 2^{Y} \longrightarrow \operatorname{ch}(X, Y)$ such that $\pi(U, V)=U \times V$. Then $(\pi \mid(A \times B), C)$ is the tensor product of Boolean algebras $A$ and $B$. In particular, $(\pi, \operatorname{ch}(X, Y))$ is the tensor product of $2^{X}$ and $2^{Y}$.

Proposition 1.5. [Has00] Consider Kripke frames $\mathrm{F}_{1}=\left(W_{1}, R_{1}\right), \mathrm{F}_{2}=\left(W_{2}, R_{2}\right)$ and their product [GS98] $\mathrm{F}_{1} \times \mathrm{F}_{2}=\left(W_{1} \times W_{2}, R_{1}^{\times}, R_{2}^{\times}\right)$. Then

(1) for any rectangle $U \times V$ we have

$$
R_{1}^{\times-1}(U \times V)=R_{1}^{-1}(U) \times V, \quad R_{2}^{\times-1}(U \times V)=U \times R_{2}^{-1}(V) ;
$$

(2) if $\left(\mathrm{F}_{1}, A_{1}\right)$ and $\left(\mathrm{F}_{2}, A_{2}\right)$ are general 1-frames, then $\left(\mathrm{F}_{1} \times \mathrm{F}_{2}, A_{1} \otimes A_{2}\right)$ is a general 2-frame. In particular, $\left(\mathrm{F}_{1} \times \mathrm{F}_{2}, \operatorname{ch}\left(W_{1}, W_{2}\right)\right)$ is a general 2-frame.

Definition 1.6. The frame $\left(\mathrm{F}_{1} \times \mathrm{F}_{2}, A_{1} \otimes A_{2}\right)$ is called the tensor product of general frames $\left(\mathrm{F}_{1}, A_{1}\right)$ and $\left(\mathrm{F}_{2}, A_{2}\right)$ :

$$
\left(\mathrm{F}_{1}, A_{1}\right) \otimes\left(\mathrm{F}_{2}, A_{2}\right):=\left(\mathrm{F}_{1} \times \mathrm{F}_{2}, A_{1} \otimes A_{2}\right) .
$$

In particular, we have the tensor product of Kripke frames $\mathrm{F}_{1} \otimes \mathrm{F}_{2}=\left(\mathrm{F}_{1} \times \mathrm{F}_{2}, \operatorname{ch}\left(W_{1}, W_{2}\right)\right)$. Tensor products of Kripke frames are also called chequered frames.

Theorem 1.7. GSS If $\left(A, \diamond_{1}\right),\left(B, \diamond_{2}\right)$ are normal 1-modal algebras, then there exists a unique 2-modal algebra structure on $A \otimes B$ such that for any $a \in A, b \in B$

$$
\diamond_{1}^{\times}(a \otimes b)=\diamond_{1} a \otimes b, \diamond_{2}^{\times}(a \otimes b)=a \otimes \diamond_{2} b .
$$

Proof. Due to the Jónsson-Tarski representation theorem [CZ96], this follows from Proposition 1.5 the operations $\diamond_{1}^{\times}$and $\diamond_{2}^{\times}$are introduced according to (1).

For classes of algebras (general frames) $\mathfrak{A}, \mathfrak{B}$, put $\mathfrak{A} \otimes \mathfrak{B}:=\{A \otimes B \mid A \in \mathfrak{A}, B \in \mathfrak{B}\}$.

Definition 1.8. The tensor product of $\operatorname{logics} \mathrm{L}_{1}$ and $\mathrm{L}_{2}$ is the $\operatorname{logic}$

$$
\mathrm{L}_{1} \otimes \mathrm{L}_{2}:=\log \left(\mathrm{Alg}\left(\mathrm{L}_{1}\right) \otimes \operatorname{Alg}\left(\mathrm{L}_{2}\right)\right) .
$$

Clearly, $\mathrm{L}_{1} \otimes \mathrm{L}_{2}=\log \left(\operatorname{GFr}\left(\mathrm{L}_{1}\right) \otimes \operatorname{GFr}\left(\mathrm{L}_{2}\right)\right)$.

The next proposition easily follows from this definition.

Proposition 1.9. 1. $\mathrm{L}_{1} \otimes \mathrm{L}_{2}$ is consistent iff $\mathrm{L}_{1}$ and $\mathrm{L}_{2}$ are consistent.

2. If $\mathrm{L}_{1}$ and $\mathrm{L}_{2}$ are consistent, then $\mathrm{L}_{1} \otimes \mathrm{L}_{2}$ is conservative over $\mathrm{L}_{1}$ and $\mathrm{L}_{2}$.

3. If $\mathrm{L}_{1} \otimes \mathrm{L}_{2}$ is consistent and Kripke complete, then $\mathrm{L}_{1}$ and $\mathrm{L}_{2}$ are Kripke complete. 


\section{Logical invariance}

This section contains some important basic properties of tensor products. The results of this section were obtained in Has00.

Theorem 2.1. Let $\mathfrak{A}, \mathfrak{A}^{\prime}, \mathfrak{B}, \mathfrak{B}^{\prime}$ be classes of 1-modal algebras, and $\log (\mathfrak{A})=\log \left(\mathfrak{A}^{\prime}\right)$, $\log (\mathfrak{B})=\log \left(\mathfrak{B}^{\prime}\right)$. Then $\log (\mathfrak{A} \otimes \mathfrak{B})=\log \left(\mathfrak{A}^{\prime} \otimes \mathfrak{B}^{\prime}\right)$.

Corollary 2.2. Let $\mathfrak{C}_{1}, \mathfrak{C}_{2}$ be classes of 1-modal algebras or general frames, $\mathrm{L}_{1}=\log \left(\mathfrak{C}_{1}\right)$, $\mathrm{L}_{2}=\log \left(\mathfrak{C}_{2}\right)$. Then $\mathrm{L}_{1} \otimes \mathrm{L}_{2}=\log \left(\mathfrak{C}_{1} \otimes \mathfrak{C}_{2}\right)$. In particular, if $\mathrm{L}_{1}$ and $\mathrm{L}_{2}$ are Kripke complete then for any classes of Kripke frames $\mathfrak{F}_{1}, \mathfrak{F}_{2}$ such that $\mathrm{L}_{i}=\log \left(\mathfrak{F}_{i}\right), i=1,2$, we have that $\mathrm{L}_{1} \otimes \mathrm{L}_{2}=\log \left(\mathfrak{F}_{1} \otimes \mathfrak{F}_{2}\right)$ is a logic of a class of chequered frames.

For classes of Kripke frames $\mathfrak{F}, \mathfrak{G}, \mathfrak{F} \times \mathfrak{G}:=\{\mathrm{F} \times \mathrm{G} \mid \mathrm{F} \in \mathfrak{F}, \mathrm{G} \in \mathfrak{G}\}$. For logics $\mathrm{L}_{1}, \mathrm{~L}_{2}$, $\mathrm{L}_{1} \times_{\text {fin }} \mathrm{L}_{2}:=\log \left(\operatorname{Fr}_{f i n}\left(\mathrm{~L}_{1}\right) \times \operatorname{Fr}_{f i n}\left(\mathrm{~L}_{2}\right)\right)$, where $\operatorname{Fr}_{f i n}(\mathrm{~L})$ denotes the class of all finite L-frames. Recall that $\mathrm{L}$ has the finite model property (the fmp, for short) if $\mathrm{L}=\log \left(\operatorname{Fr}_{\text {fin }}(\mathrm{L})\right)$. A logic $\mathrm{L}_{1} \times \mathrm{L}_{2}$ has the product fmp if $\mathrm{L}_{1} \times \mathrm{L}_{2}=\mathrm{L}_{1} \times f$ in $\mathrm{L}_{2}$.

Corollary 2.3. 1. For any $\mathrm{L}_{1}, \mathrm{~L}_{2}, \mathrm{~L}_{1} \otimes \mathrm{L}_{2}=\log \left(\left(\mathrm{F}_{\mathrm{L}_{1}}, A_{\mathrm{L}_{1}}\right) \otimes\left(\mathrm{F}_{\mathrm{L}_{2}}, A_{\mathrm{L}_{2}}\right)\right)$, where $\mathrm{F}_{\mathrm{L}}$ denotes the canonical frame of a logic $\mathrm{L}$, and $\left(\mathrm{F}_{\mathrm{L}}, A_{\mathrm{L}}\right)$ denotes its general canonical frame.

2. If $\mathrm{L}_{1}, \mathrm{~L}_{2}$ are canonical, then $\mathrm{L}_{1} \otimes \mathrm{L}_{2}=\log \left(\mathrm{F}_{\mathrm{L}_{1}} \otimes \mathrm{F}_{\mathrm{L}_{2}}\right)$.

3. If $\mathrm{L}_{1}, \mathrm{~L}_{2}$ are Kripke complete, then $\mathrm{L}_{1} \times \mathrm{L}_{2} \subseteq \mathrm{L}_{1} \otimes \mathrm{L}_{2} \subseteq \mathrm{L}_{1} \times$ fin $\mathrm{L}_{2}$.

4. Let $\mathrm{L}_{1}, \mathrm{~L}_{2}$ be Kripke complete logics. $\mathrm{L}_{1} \times \mathrm{L}_{2}$ has the product fmp iff

$$
\mathrm{L}_{1} \times \mathrm{L}_{2}=\mathrm{L}_{1} \otimes \mathrm{L}_{2}=\mathrm{L}_{1} \times_{f i n} \mathrm{~L}_{2}
$$

it follows that if $\mathrm{L}_{1} \times \mathrm{L}_{2}$ has the product fmp, then for any $\mathfrak{F}_{i}$ such that $\mathrm{L}_{i}=\log \left(\mathfrak{F}_{i}\right)$, $i=1,2$, we have $\mathrm{L}_{1} \times \mathrm{L}_{2}=\log \left(\mathfrak{F}_{1} \times \mathfrak{F}_{2}\right)$.

5. Suppose $\mathrm{L}_{1}$ and $\mathrm{L}_{2}$ have the fmp. Then $\mathrm{L}_{1} \otimes \mathrm{L}_{2}=\mathrm{L}_{1} \times{ }_{\text {fin }} \mathrm{L}_{2}$, and

$$
\mathrm{L}_{1} \times \mathrm{L}_{2} \text { has the product fmp iff } \mathrm{L}_{1} \times \mathrm{L}_{2}=\mathrm{L}_{1} \otimes \mathrm{L}_{2} \text {. }
$$

It follows that usually modal and tensor products are different. In fact, modal products of logics with the fmp in many cases do not have the product fmp GKWZ03. Rare exceptions are $\mathbf{K} \times \mathbf{K}$ and $\mathbf{S 5} \times \mathbf{S 5}$ GS98, GKWZ03. Further on we describe some other cases.

\section{$3 \quad$ Filtrations of chequered models}

Recall the standard construction of filtration of Kripke models [CZ96].

Definition 3.1. Let $\mathrm{M}=(W, R, \theta)$ be a model, $\Phi$ a set of formulas.

Consider the equivalence relation $\sim_{\Phi}:=\left\{\left(x, x^{\prime}\right) \mid \forall \varphi \in \Phi\left(\mathrm{M}, x \vDash \varphi \Leftrightarrow \mathrm{M}, x^{\prime} \vDash \varphi\right)\right\}$.

$[x]$ denotes the equivalence class of $x$ w.r.t. $\sim_{\Phi}$. Consider relations $R_{\min }^{\mathrm{M}, \Phi}$ and $R_{\max }^{\mathrm{M}, \Phi}$ on the quotient set $W / \sim_{\Phi}$ :

$$
\begin{aligned}
& R_{\min }^{\mathrm{M}, \Phi}:=\left\{([x],[y]) \mid \exists x^{\prime} \in[x] \exists y^{\prime} \in[y] \quad x^{\prime} R y^{\prime}\right\}, \\
& R_{\max }^{\mathrm{M}, \Phi}:=\{([x],[y]) \mid \forall \varphi(\diamond \varphi \in \Phi \& \mathrm{M}, y \vDash \varphi \Rightarrow \mathrm{M}, x \vDash \diamond \varphi)\} .
\end{aligned}
$$

They are called the minimal and the maximal filtrating relations. A model $\overline{\mathrm{M}}=\left(W / \sim_{\Phi}, \bar{R}, \bar{\theta}\right)$ is called a filtration of $\mathrm{M}$ through $\Phi$, if for any $p \in \Phi \bar{\theta}(p)=\{[x] \mid \mathrm{M}, x \vDash p\}$, and $R_{\text {min }}^{\mathrm{M}, \Psi} \subseteq \bar{R} \subseteq$ $R_{\max }^{\mathrm{M}, \Psi}$. 
For models $\mathrm{M}_{1}, \mathrm{M}_{2}$, the notation $\mathrm{M}_{1}, x \sim_{\Phi} \mathrm{M}_{2}, y$ means $\forall \varphi \in \Phi\left(\mathrm{M}_{1}, x \vDash \varphi \Leftrightarrow \mathrm{M}_{2}, y \vDash \varphi\right)$.

Lemma 3.2 (Filtration Lemma). [CZ96] Consider a set of formulas $\Phi$ closed under subformulas. If $\overline{\mathrm{M}}$ is a filtration of $\mathrm{M}$ through $\Phi$, then $\mathrm{M}, x \sim_{\Phi} \overline{\mathrm{M}},[x]$ for any $x$ in $\mathrm{M}$.

Definition 3.3. Gab72 A logic L admits filtration if it is Kripke complete and for any Lframe $(W, R)$, for any model $\mathrm{M}=(W, R, \theta)$, and for any finite set of formulas $\Psi$ closed under subformulas, there exists a filtration $(\bar{W}, \bar{R}, \bar{\eta})$ of $\mathrm{M}$ through $\Psi$ such that $(\bar{W}, \bar{R})$ is an L-frame.

It is well known that many logics admit filtration (for example, $\mathbf{K}, \mathbf{K} 4, \mathbf{T}, \mathbf{S 4}, \mathbf{S 5}$ ).

It follows that if a logic $\mathrm{L}$ admits filtration, then it has the fmp. Moreover, to check the L-satisfiability of a formula $\varphi$, it is sufficient to consider L-frames of cardinality not greater then $2^{\# \varphi}$, where $\# \varphi$ denotes the cardinality of $\operatorname{sub}(\varphi), \operatorname{sub}(\varphi)$ denotes the set of all subformulas of $\varphi$. Our aim is to formulate an analogous result for chequered frames.

Definition 3.4. Consider Kripke frames $\mathrm{F}_{1}, \mathrm{~F}_{2}, \mathrm{~F}_{i}=\left(W_{i}, R_{i}\right)$, a model $\mathrm{M}=\left(\mathrm{F}_{1} \times \mathrm{F}_{2}, \theta\right)$, and a set of formulas $\Phi$. Consider relations $\sim_{i}$ on $W_{i}$ :

$$
\begin{aligned}
& \sim_{1}:=\left\{\left(x, x^{\prime}\right) \mid(x, y) \sim_{\Phi}\left(x^{\prime}, y\right) \text { for all } y \in W_{2}\right\} \\
& \sim_{2}:=\left\{\left(y, y^{\prime}\right) \mid(x, y) \sim_{\Phi}\left(x, y^{\prime}\right) \text { for all } x \in W_{1}\right\} .
\end{aligned}
$$

The pair $\left(\sim_{1}, \sim_{2}\right)$ is called the $\Phi$-granulation of M. Obviously, $\sim_{i}$ is an equivalence relation on $W_{i}$.

Proposition 3.5. Consider a model $\mathrm{M}=\left(\left(W_{1}, R_{1}\right) \otimes\left(W_{2}, R_{2}\right), \theta\right)$, a finite set of formulas $\Phi$, and the $\Phi$-granulation $\left(\sim_{1}, \sim_{2}\right)$. Then the quotient sets $W_{1} / \sim_{1}$ and $W_{2} / \sim_{2}$ are finite.

Lemma 3.6. Suppose L admits filtration, F, G are Kripke frames, $\mathrm{F} \vDash \mathrm{L}, \varphi$ is true at some point in a chequered model $\mathrm{M}=(\mathrm{F} \otimes \mathrm{G}, \theta),\left(\sim_{1}, \sim_{2}\right)$ is the $\operatorname{sub}(\varphi)$-granulation of $\mathrm{M}$. Then there exists a Kripke frame $\overline{\mathrm{F}}$ such that $\overline{\mathrm{F}} \vDash \mathrm{L}, \varphi$ is satisfiable in $\overline{\mathrm{F}} \times \mathrm{G}$, and the cardinality of $\overline{\mathrm{F}}$ is not greater than

$$
2^{\# \varphi \cdot\left|W_{2} / \sim_{2}\right|} .
$$

Unlike the usual filtration technique, this lemma does not estimate the size of a countermodel, since the upper bound depends on $W_{2} / \sim_{2}$. However, in some cases it implies decidability results for tensor and modal products.

Theorem 3.7. If $\mathrm{L}_{1}$ is Kripke complete, $\mathrm{L}_{2}$ is tabular, then $\mathrm{L}_{1} \times \mathrm{L}_{2}=\mathrm{L}_{1} \otimes \mathrm{L}_{2}$.

Proof. $\mathrm{L}_{1} \times \mathrm{L}_{2}$ is complete with respect to the class $\mathfrak{C}=\left\{\mathrm{F} \times \mathrm{G} \mid \mathrm{F} \vDash \mathrm{L}_{1}, \mathrm{G} \vDash \mathrm{L}_{2}, \mathrm{G}\right.$ is rooted $\}$, see e.g. GKWZ03. If $\mathrm{G} \vDash \mathrm{L}_{2}$ and $\mathrm{G}$ is rooted, then by tabularity of $\mathrm{L}_{2}, \mathrm{G}$ is finite CZ96. So all frames in the class $\mathfrak{C}$ are chequered, and $\mathrm{L}_{1} \times \mathrm{L}_{2}=\mathrm{L}_{1} \otimes \mathrm{L}_{2}$.

Hence we readily obtain the following properties of modal products with tabular logics.

Corollary 3.8. For a class of frames $\mathfrak{F}$, and a finite frame $\mathrm{G}, \log (\mathfrak{F}) \times \log (\mathrm{G})=\log (\mathfrak{F} \times\{\mathrm{G}\})$.

Corollary 3.9. The modal product of tabular logics is tabular: if $\mathrm{F}$ and $\mathrm{G}$ are finite, then

$$
\log (\mathrm{F}) \times \log (\mathrm{G})=\log (\mathrm{F} \times \mathrm{G}) .
$$

Theorem 3.10. Suppose $\mathrm{L}_{1}$ admits filtration, $\mathrm{L}_{2}$ is tabular. Then:

1. $\mathrm{L}_{1} \times \mathrm{L}_{2}$ has the exponential product fmp;

2. if the finite frame problem is decidable for $\mathrm{L}_{1}$, then $\mathrm{L}_{1} \times \mathrm{L}_{2}$ is decidable.

Proof. Let $\mathrm{L}_{2}=\log (\mathrm{G})$ for a finite $\mathrm{G}$ of size $n$. By Lemma 3.6, $\varphi$ is $\mathrm{L}_{1} \times \mathrm{L}_{2}$-satisfiable iff $\varphi$ is satisfiable in a frame $\mathrm{F} \times \mathrm{G}$, where $\mathrm{F} \vDash \mathrm{L}_{1}$ and the size of $\mathrm{F}$ is not greater than $2^{\# \varphi \cdot n}$. 


\section{Open questions}

The above considerations can be easily transferred to the polymodal case. In particular, logical invariance holds for the polymodal case. One of the corollaries is associativity of the tensor products. Note that this question for modal products is open, see e.g. [Kur07, p. 877].

Still there are many open questions about tensor products of modal logics, for example:

1. Does Kripke completeness transfer from $\mathrm{L}_{1}$ and $\mathrm{L}_{2}$ to $\mathrm{L}_{1} \otimes \mathrm{L}_{2}$ ?

2. Do there exist $\mathrm{L}_{1}, \mathrm{~L}_{2}$ such that $\mathrm{L}_{1} \times \mathrm{L}_{2}=\mathrm{L}_{1} \otimes \mathrm{L}_{2}$, but $\mathrm{L}_{1} \times \mathrm{L}_{2}$ lacks the product fmp and $\mathrm{L}_{1}, \mathrm{~L}_{2}$ are non-tabular?

3. Do there exist $\mathrm{L}_{1}, \mathrm{~L}_{2}$ such that $\mathrm{L}_{1} \times \mathrm{L}_{2}$ is undecidable, but $\mathrm{L}_{1} \otimes \mathrm{L}_{2}$ is decidable?

4. Do there exist $\mathrm{L}_{1}, \mathrm{~L}_{2}$ such that $\mathrm{L}_{1} \times \mathrm{L}_{2}$ is not finitely axiomatizable, but $\mathrm{L}_{1} \otimes \mathrm{L}_{2}$ is finitely axiomatizable?

5. Suppose that $\mathrm{L}_{1}$ is decidable, $\mathrm{L}_{2}$ is tabular; is $\mathrm{L}_{1} \otimes \mathrm{L}_{2}\left(=\mathrm{L}_{1} \times \mathrm{L}_{2}\right)$ decidable?

\section{References}

[AM69] M. Atiyah and I. Macdonald. Introduction to commutative algebra. Addison-WesleyLongman, 1969.

[CZ96] A. Chagrov and M. Zakharyaschev. Modal logic. Oxford University Press, 1996.

[Gab72] D. Gabbay. General filtration method for modal logic. Journal of Philos. Logic, 1:29-34, 1972.

[GKWZ03] D. Gabbay, A. Kurucz, F. Wolter, and M. Zakharyaschev. Many-dimensional modal logics: theory and applications, volume 148 of Studies in Logic and the Foundations of Mathematics. Elsevier, 2003.

[GS98] D. Gabbay and V. Shehtman. Products of modal logics, part 1. Logic Journal of the IGPL, 6(1):73-146, 1998.

[GSS] D. Gabbay, I. Shapirovsky, and V. Shehtman. Products of modal logics and tensor products of modal algebras. In preparation.

[Has00] Y. Hasimoto. Normal products of modal logics. In Frank Wolter, Heinrich Wansing, Maarten de Rijke, and Michael Zakharyaschev, editors, Advances in Modal Logic, pages 241-255. World Scientific, 2000.

[Kur07] A. Kurucz. Combining modal logics. In Handbook of Modal Logic, volume 3 of Studies in Logic and Practical Reasoning, pages 869-924. Elsevier, 2007. 